Published in final edited form as:

J AAPOS. 2003 February ; 7(1): 1-6.

\title{
Extraocular Muscle Force Generation after Ricin-mAb35 Injection: Implications for Strabismus Treatment
}

\author{
Stephen P. Christiansen, MD ${ }^{\mathrm{a}, \mathrm{b}}$, Bryan A. Becker, MS ${ }^{\mathrm{a}}$, Paul A. laizzo, PhD ${ }^{\mathrm{C}}$, , and Linda K. \\ McLoon, PhDa \\ a Department of Ophthalmology, University of Minnesota, Minneapolis \\ b Department of Pediatrics, University of Minnesota, Minneapolis \\ c Department of Anesthesiology, University of Minnesota, Minneapolis \\ $\mathrm{d}$ Department of Physiology, University of Minnesota, Minneapolis
}

\section{Abstract}

Purpose: Ricin-mAb35 is an immunotoxin targeted against skeletal muscle. Previously, we have shown that injection of ricin-mAb35 into rabbit extraocular muscle results in long-term muscle loss, and we have proposed this as a potential treatment for strabismus. In this study, we assessed the effects of ricin-mAb35 injection on extraocular muscle force generation.

Methods: Ricin-mAb35, $0.2 \mu \mathrm{g} / \mathrm{kg}$ in a volume of $0.1 \mathrm{~mL}$, was injected into 1 superior rectus muscle in 16 adult rabbits. The contralateral superior rectus was injected with an equal volume of normal saline. Muscle force generation was assessed in vivo at 1, 6, and 12 weeks. Isometric lengthtension curves were developed. Single-twitch tension, peak tetanic force generation, and fatigue rate were determined at optimal preload. Data from treated and control muscles were compared with the paired $t$ test.

Results: Force generation declined in ricin-mAb35 treated muscles at each postinjection interval. At 12 weeks, mean tetanic tension $(200 \mathrm{~Hz})$ in treated muscles was $13.8 \mathrm{mN} / \mathrm{cm}^{3}$ compared with $27.7 \mathrm{mN} / \mathrm{cm}^{3}$ in saline-injected controls $(P=.02)$, a reduction of $50 \%$. Single-twitch tension at 12 weeks was reduced $33 \%$ compared to controls $(P=.04)$. Similar effects were noted at 1 and 6 weeks. Fatigue rate was not greater in treated muscles at any postinjection intervals.

Conclusions: Injection of ricin-mAb35 results in sustained weakness in extraocular muscle, although additional studies will be required to determine the duration of physiologic effect. These results confirm our histological analysis and suggest that ricin-mAb35 may be a more long-term alternative to botulinum toxin $\mathrm{A}$ for the treatment of strabismus.

Ricin-mAb35 is an immunotoxin composed of the ribosomal poison ricin attached to a monoclonal antibody mAb35, which binds acetylcholine receptors on skeletal muscle. RicinmAb35, therefore, has specific intracellular cytotoxic activity against skeletal muscle, ${ }^{1}$ including extraocular muscle. ${ }^{2,3}$ In earlier studies, we have shown that injections of ricin$\mathrm{mAb35}$ into superior rectus muscles in rabbits results in rapid focal muscle fiber loss. Total muscle cross-sectional area and muscle fiber number remain decreased through 105 days postinjection and then recover slowly by muscle regeneration. Even at 1 year, however, there

Reprint requests: Stephen P. Christiansen, MD, Department of Ophthalmology, University of Minnesota, MMC 493, 420 Delaware St, SE, Minneapolis, MN 55455-0501..

Presented at the 28th Annual Meeting of the American Association for Pediatric Ophthalmology and Strabismus, Seattle, Washington, March 2002.

Supported by an unrestricted grant from Research to Prevent Blindness, Inc, New York, New York. 
are persistent changes in myosin isoform composition and fiber morphometry in treated muscles when compared with saline-injected controls. The immunotoxin appears to be welltolerated, with a minimal and short-lived inflammatory response, and has no demonstrable effects on other orbital tissues. Treated animals show no evidence of systemic or local toxicity. 2,3

We have proposed that ricin-mAb35 may be useful in the treatment of strabismus. An important requisite of any agent being suggested as potentially useful for the treatment of strabismus is that its effects last sufficiently long that motor and sensory adaptation can occur, allowing longterm changes in the rotational position of the globe. A serious drawback to the use of botulinum toxin type A for most types of strabismus is its relatively short duration of action. ${ }^{4}$ This often necessitates reinjection ${ }^{5}$ or conversion to incisional surgery. In contrast, previous work in limb muscle indicates that local infusion of ricin-mAb35 results in sustained weakness of treated muscles. ${ }^{1}$ In this study, we extend these observations to extraocular muscle and measure the early effects of ricin-mAb35 injection on force generation and fatigability.

\section{MATERIALS AND METHODS}

All experimental procedures were reviewed and preap-proved by the Institutional Animal Care and Use Committee at the University of Minnesota, and conform to published guidelines of the National Institutes of Health (NIH) for use of animals in research. Adult New Zealand white rabbits were obtained from Birchwood Farms (Red Wing, Minn) and housed with Research Animal Resources at the University of Minnesota.

\section{Surgical Injection}

Ricin-mAb35 was obtained from the laboratories of Richard J. Youle, PhD, at the NIH, Bethesda, Md. Hott et a ${ }^{1}$ have previously described the preparation and purification of the immunotoxin. Our earlier histological studies indicated that a dose of a tenth the maximally tolerated dose (MTD) for mice, or $0.2 \mu \mathrm{g} / \mathrm{kg}$, achieved the most reproducible effect in rabbit extraocular muscle, ${ }^{2}$ and this dose was used throughout the current experiments.

Sixteen rabbits were anesthetized with an intramuscular injection of ketamine and xylazine, $10 \mathrm{mg} / \mathrm{kg}$ and $2 \mathrm{mg} / \mathrm{kg}$, respectively. Proparacaine drops were placed in the conjunctival culde-sac to reduce the blink reflex. The superior rectus muscles of each rabbit were randomly assigned to treatment and control groups. These muscles were chosen because of their proximity to the limbus, the ease of surgical exposure, and accessibility for physiological testing. Using aseptic technique, a superior conjunctival peritomy was performed, and the conjunctiva was retracted, exposing the distal superior rectus muscle. Under direct observation, 1 superior rectus muscle of each rabbit was injected with ricin-mAb35, diluted with sterile isotonic saline to a tenth the MTD in a volume of $100 \mu \mathrm{L}$. The needle was left in place for 30 seconds to allow diffusion of the toxin into the muscle and to minimize leakage into the orbit. An identical procedure was then performed in the opposite orbit, substituting an equal volume of normal saline for the toxin. A combination antibiotic/steroid ointment (tobramycin/ dexamethasone) was applied to each eye. The animals recovered in separate cages with ample food and water. They were monitored daily for inflammatory orbital changes, evidence of discomfort, irritability, poor appetite, etc. Acetaminophen was administered in the animals' water if signs of discomfort were noted.

\section{Physiological Testing}

In situ muscle force measurements were taken from the superior rectus muscle in both orbits at 1-, 6-, and 12-week postinjection intervals. Each rabbit was anesthetized with intramuscular injections of ketamine, $50 \mathrm{mg} / \mathrm{kg}$, and xylazine, $10 \mathrm{mg} / \mathrm{kg}$, respectively. After endotracheal 
intubation, inhalational anesthesia was initiated with isoflurane (up to 5\%). The rabbits were ventilated continuously and were monitored with a pulse oximeter. Core body temperature was monitored with a rectal probe and maintained within the normal range using a heating pad. A 3 -channel electrocardiogram was used to monitor heart rate. The animals' heads were fixed in a stereotactic frame and secured with a nose clamp to prevent any head movement.

The upper eyelids and the superior bulbar conjunctiva were removed, exposing the superior rectus muscles of both orbits. The insertional tendons were transected from the globe and attached to isometric force transducers with 4-0 silk. The force transducers were mounted on isometric tension clamps with the line of force perpendicular to the animals' midline and elevated slightly above the horizontal plane of the animals' head. This allowed adjustments in the resting length of the muscles with minimal drag from the globe. Generated force in grams was recorded using LabVIEW software (National Instruments Corporation, Austin, Tex). Grams are converted to $\mathrm{mN} / \mathrm{cm}^{3}$ by using the constant $1.056 \mathrm{grams} / \mathrm{cm}^{3}$, the muscle weight, and the muscle length to determine the muscle volume.

Bipolar platinum contact electrodes were positioned on the distal third of the muscles, near the insertional tendons, to avoid indirect stimulation of the muscles by terminal nerves or the endplate region. Both treated and control superior rectus muscles were tested simultaneously. The supramaximal stimulation intensity was determined by increasing voltage until maximal contraction was achieved using square-wave pulses of 0.4 millisecond duration. Isometric length-tension curves were established by stimulating each muscle at supramaximal intensity ( $\geq 5 \mathrm{~V}, 0.4$ milliseconds) while varying the preload (resting length) over a range of $0.15 \mathrm{~g}$ to $10.0 \mathrm{~g}$. The optimal preload was determined by incrementally increasing the resting muscle length to achieve maximum isometric twitch force, allowing 60 seconds of rest between stimuli. All further testing was then performed at optimal preload with supramaximal stimulus intensities.

Tension development was measured for single, double, triple, and quadruple pulses ( 0.4 millisecond pulse duration) with 2 minutes rest between stimuli. The muscles then were stimulated at 10,20, 40,100,150, and $200 \mathrm{~Hz}$ ( 250 millisecond train duration) with 4 minutes of rest between stimuli. Fatigability was tested by stimulating the muscles at $100 \mathrm{~Hz}(250$ millisecond train duration) every 10 seconds for 1600 seconds or until there was a $50 \%$ reduction in generated force. Data from treated and control muscles were pooled at each postinjection interval and compared with the paired $t$ test. A $P$ value less than or equal to .05 was considered statistically significant.

At the completion of testing, the animals were killed with an overdose of intravenous barbiturate anesthesia. The superior rectus muscles of each eye were removed, weighed, measured, and prepared for histological examination.

\section{Histology}

The superior rectus muscles from each orbit were embedded in tragacanth gum, quick-frozen in 2-methylbutane chilled to a slurry in liquid nitrogen, and then serially cross-sectioned at 12 $\mu \mathrm{m}$ on a cryostat. The sections were stained with hematoxylin and eosin, and the area of ricinmAb35 induced damage was assessed using a Bioquant Image Analysis System (BIOQUANTR\&M Biometrics, Inc, Nashville, Tenn).

\section{RESULTS}

Injection of ricin-mAb35, $0.2 \mu \mathrm{g} / \mathrm{kg}$ (a tenth MTD) into 1 superior rectus muscle appeared to be well tolerated by all experimental animals. No animal exhibited local or systemic signs of 
toxicity. The immunotoxin-treated muscles did not show excessive scarring or adhesion when compared to the saline-injected control muscles at the time of physiological testing.

No obvious alteration in the rotational position of the globe was seen in either the saline or ricin-mAb35 treated eyes. It was not uncommon, however, to see mild upper lid ptosis in the first 24 to 48 hours following ricin-mAb35 injection.

Ricin-mAb35-treated muscles showed a substantial decrement in both single twitch and tetanic tension development that persisted through the 12-week postinjection interval (Tables 1, 2, and Figure 1). Even at 12 weeks, single twitch and tetanic $(200 \mathrm{~Hz})$ tensions were at $67 \%$ and $50 \%$ of saline-treated control muscles, respectively. Force generation appeared to reach a nadir at 6 weeks postinjection, however, with evidence of some recovery at 12 weeks (Tables 1 and 2). Both treated and control superior rectus muscles proved remarkably resilient to fatigue testing at all postinjection intervals with little to no decline in generated tension even after extended periods of time, stimulating at relatively high frequencies (Figure 2).

Superior rectus muscles removed after physiological testing all showed evidence of trauma including a mild inflammatory infiltrate, edema, and myofiber injury. At the 6- and 12-week postinjection intervals, areas of muscle fiber hypertrophy and myofibers with central nuclei suggested ongoing muscle regeneration (Figure 3).

\section{DISCUSSION}

The clinical feasibility and use of pharmacological treatment of strabismus has been proven with botulinum toxin A. Biochemical denervation of extraocular muscle with this potent toxin alters the rotational position of the globe and may satisfactorily restore single binocular vision. Treatment with botulinum toxin A is less invasive, less likely to disrupt anterior segment circulation, less time-consuming, and less likely to create scarring than traditional incisional procedures. In addition, it maintains important anatomical parameters in the treated muscle, such as its arc of contact with the globe and its relationship with its soft-tissue pulley. ${ }^{6}$ The only significant drawback to the clinical use of botulinum toxin $\mathrm{A}$ is its relatively brief duration of action. ${ }^{4}$ However, given the choice between reinjection and the often more predictable results of strabismus surgery, many patients and surgeons will elect surgery. This highlights the need for studies of new agents that build on the groundwork of botulinum toxin A, that have a similar safety profile, and yet are longer-lasting.

Potent biological toxins, linked to monoclonal antibodies against specific cell-surface antigens or receptors are called immunotoxins. These toxins permit selective targeting of tissues to accomplish a clinical effect. The vast majority of work with immunotoxins, to date, has been in the area of hematology and oncology. ${ }^{7-10}$ However, a recent study of ricin, linked to a monoclonal antibody against the nicotinic acetylcholine receptor on skeletal muscle fibers (mAb35) suggests that this new immunotoxin may have a use in the treatment of certain limb muscle dystonias. It produces selective destruction of muscle fibers and muscle weakness at safe doses and appears to last longer than botulinum toxin A. ${ }^{1}$ Our earlier histological studies in extraocular muscle showed that treatment with ricin-mAb35 resulted in sustained muscle fiber loss with more subtle changes lasting even to a year postinjection. ${ }^{2,3}$

The results of the current study corroborate our histological observations and indicate that ricinmAb35 substantially decreases extraocular muscle force generation at least through 12 weeks postinjection. Generated tetanic force remained reduced by $50 \%$ at 12 weeks, although there are early indications that force generation was beginning to recover at this interval. The duration of treatment effect remains to be determined in future studies, but will be crucial to the success of this agent if it is to be used in the treatment of strabismus. A treatment effect must last long enough that sensory and motor adaptation can occur to create a permanent change in the 
rotational position of the globe. However, our results confirm the observations of Hott et al ${ }^{1}$ in limb muscle, that the reduction in force generation following ricin-mAb35 injection persists beyond the period of effectiveness of botulinum toxin A.

In contrast to botulinum toxin, where functional recovery occurs by means of axonal sprouting and formation of new neuromuscular junctions, ${ }^{11}$ recovery from a cytotoxic injury, such as that induced by ricin-mAb35, occurs by means of muscle regeneration. Histological evaluation of tested muscles in this study (Figure 3 ) and in our previous studies ${ }^{3}$ showed hypertrophic muscle fibers with central nuclei indicating regenerating myofibers. Muscle regeneration is normally a slow process. Other investigators have shown that even morphologically normal appearing muscles produce subnormal tetanic tensions 90 days after a muscle injury. ${ }^{12,13}$ This slow regenerative process may explain the longer duration of action seen following ricinmAb35 treatment.

Despite the reduction in force generation, treated superior rectus muscles were remarkably fatigue-resistant at all postinjection intervals. Even with repetitive and sustained stimulation lasting greater than 25 minutes, we did not observe any decline in force generation from baseline in either treated or control muscles. Extraocular muscle blood flow and potential oxidative capacity is higher than in any other skeletal muscle, making these among the fastest and most fatigue-resistant of the mammalian muscles. ${ }^{14}$ Our previous histological studies have shown that ricin-mAb35 injection results is a discrete cytotoxic lesion with good preservation of intact myofibers peripheral to the injection site. ${ }^{3}$ The immunotoxin reduces muscle mass and myofiber number, but remaining muscle appears physiologically intact.

This study does not address the effectiveness of ricin-mAb35 in altering the rotational position of the globe. Botulinum toxin A presumably works in strabismus by decreasing extraocular muscle force generation sufficiently that the resting tone of the antagonist rotates the globe out of the field of action of the injected muscle. ${ }^{4}$ The only study, to date, comparing ricin-mAb35 and botulinum toxin $\mathrm{A}$ in rats used rotorod exercise tolerance to assess limb muscle strength. In that series of experiments, ricin-mAb35 appeared 30 times more potent than botulinum toxin A and produced a dramatically longer response time. ${ }^{1}$ Comparative studies will be needed in extraocular muscle, but this preliminary work suggests that ricin-mAb35 is sufficiently potent to alter eye alignment.

Safety and toxicity concerns about new toxins are valid and were not directly addressed in this study. In this and in our previous studies with ricin-mAb35, no animal exhibited evidence of systemic toxicity, and no animal died as a direct consequence of treatment. Local toxic effects appeared limited to a brief inflammatory response isolated to the treated muscle. Even within the muscle, the toxic effects of ricin-mAb35 were limited to mature myofibers. Nerve and vascular tissue were unaffected. ${ }^{2}$ Therein lays the benefit of immunotoxins such as ricinmAb35. Despite the known toxicity of free ricin, this potent toxin when bound to a monoclonal antibody against acetylcholine receptors affects the target muscle only. Distant effects on limb muscle following extraocular muscle injection will need to be assessed. However, the highly focal lesion produced within injected extraocular muscles suggests that limb muscle myotoxicity will be minimal.

Although ricin-mAb35 shows promise for treatment of strabismus, a number of issues must be addressed before it can be recommended for use in humans. Most important of these is the period of muscle weakness that is optimal for treatment of strabismus. Although we argue that the duration of action of botulinum toxin A is too short, it is conceivable that an agent may have too long a duration of action. For example, long-term extraocular muscle weakness may result in persistent consecutive strabismus that might be especially undesirable in children at risk for amblyopia. Furthermore, leakage of immunotoxin from the injected muscle may induce 
unwanted and prolonged effects in nontarget muscles. More long-term physiologic studies and additional testing planned for nonhuman primates will help answer some of these important questions.

The results of the current study correlate well with our earlier histological observations. The prolonged myopathic effect observed following injection of ricin-mAb35 is reflected in sustained weakness of the treated extraocular muscle, at least to 12 weeks postinjection. While additional questions remain, this preliminary work suggests that ricin-mAb35 may be useful as a more long-term alternative to botulinum toxin $\mathrm{A}$ in the treatment of strabismus and other neuromuscular diseases.

\section{References}

1. Hott JS, Dalakas MC, Sung C, Hallett M, Youle RJ. Skeletal muscle-specific immunotoxin for the treatment of focal muscle spasm. Neurology 1998;50:485-91. [PubMed: 9484377]

2. Christiansen S, Sandnas A, Prill R, Youle RJ, McLoon LK. Acute effects of the skeletal muscle-specific immunotoxin, ricin-mAb35, on extraocular muscles of rabbits. Invest Ophthalmol Vis Sci 2000;41:3402-9. [PubMed: 11006231]

3. Christiansen SP, Peterson D, To T, Youle R, McLoon L. Long-term effects of ricin-mAb35 on extraocular muscles of rabbits: potential treatment for strabismus. Invest Ophthalmol Vis Sci 2002;43:679-85. [PubMed: 11867584]

4. Scott AB. Botulinum toxin injection into extraocular muscles as an alternative to strabismus surgery. Ophthalmology 1980;87:1044-9. [PubMed: 7243198]

5. Tejedor J, Rodriguez JM. Long-term outcome and predictor variables in the treatment of acquired esotropia with botulinum toxin. Invest Ophthalmol Vis Sci 2001;42:2542-6. [PubMed: 11581195]

6. Demer JL, Oh SY, Poukens V. Evidence for active control of rectus extraocular muscle pulleys. Invest Ophthalmol Vis Sci 2000;41:1280-90. [PubMed: 10798641]

7. Vallera DA, Ash R, Zanjani E, Kersey J, LeBien T, Beverley P, et al. Anti-T-cell reagents for human bone marrow transplantation: ricin linked to three monoclonal antibodies. Science 1983;222:512-5. [PubMed: 6353579]

8. Filipovich AH, Vallera D, Youle RJ, Quinones R, Neville D, Kersey JH. Ex-vivo treatment of donor bone marrow with anti-T-cell im-munotoxins for prevention of graft-versus-host disease. Lancet 1984;1:469-72. [PubMed: 6142206]

9. Laske DW, Oldfield E, DeVroom H, Sung C, Dedrick R, Simon T, et al. Intraventricular immunotoxin therapy for leptomeningeal neo-plasia. Neurosurgery 1997;41:1039-49. [PubMed: 9361057]

10. Laske DW, Youle RJ, Oldfield E. Tumor regression with regional distribution of the targeted toxin TF-CRM107 in patients with malignant brain tumors. Nat Med 1997;3:1362-8. [PubMed: 9396606]

11. Holds JB, Alderson K, Fogg SG, Anderson RL. Motor nerve sprouting in human orbicularis oculi muscle after botulinum A injection. Invest Ophthalmol Vis Sci 1990;31:964-7. [PubMed: 2335457]

12. Louboutin JP, Fichter-Gegnepain V, Noireaud J. External calcium dependence of extensor digitorum longus muscle contractibility during bupivacaine-induced regeneration. Muscle Nerve 1996;19:9941002. [PubMed: 8756165]

13. Rosenblatt JD, Woods RI. Hypertrophy of rat extensor digitorum longus muscle injections with bupivacaine. A sequential histochem-ical, immunohistochemical, histological, and morphometric study. J Anat 1992;181:11-27. [PubMed: 1294558]

14. Porter JD, Baker RS, Ragusa RJ, Brueckner JK. Extraocular muscles: basic and clinic aspects of structure and function. Surv Ophthalmol 1995;39:451-84. [PubMed: 7660301] 


\section{A. 1 Week Post-Injection}

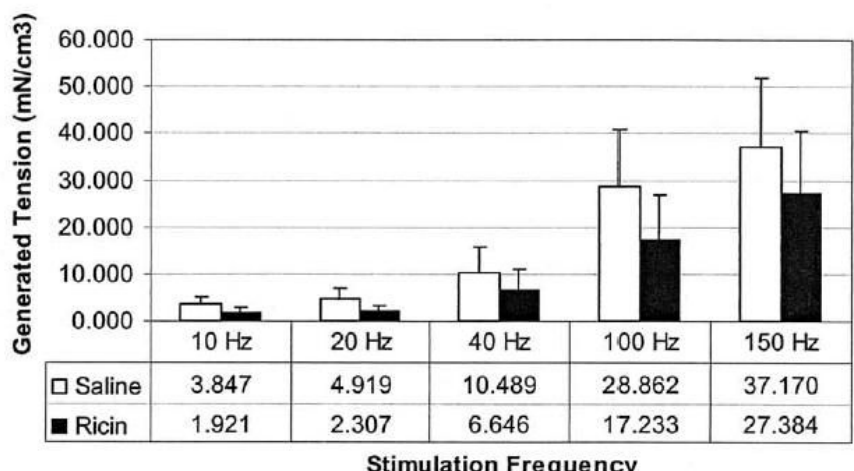

B. 6 Weeks Post-Injection

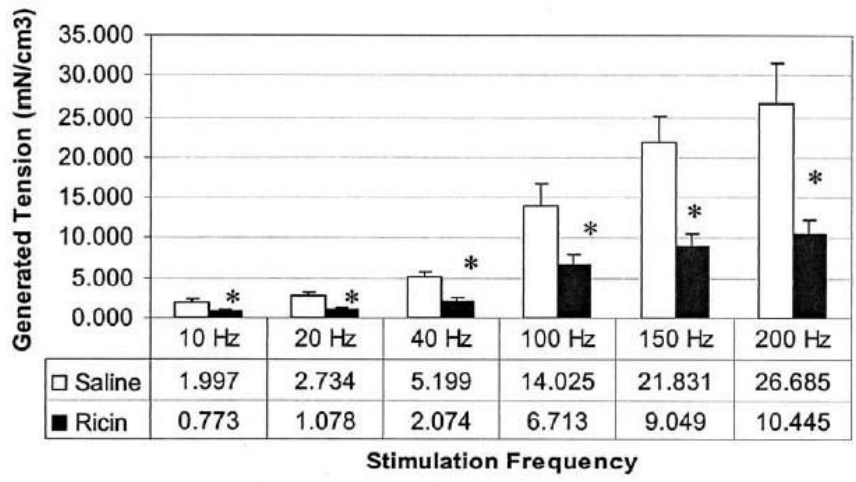

C. 12 Weeks Post-Injection

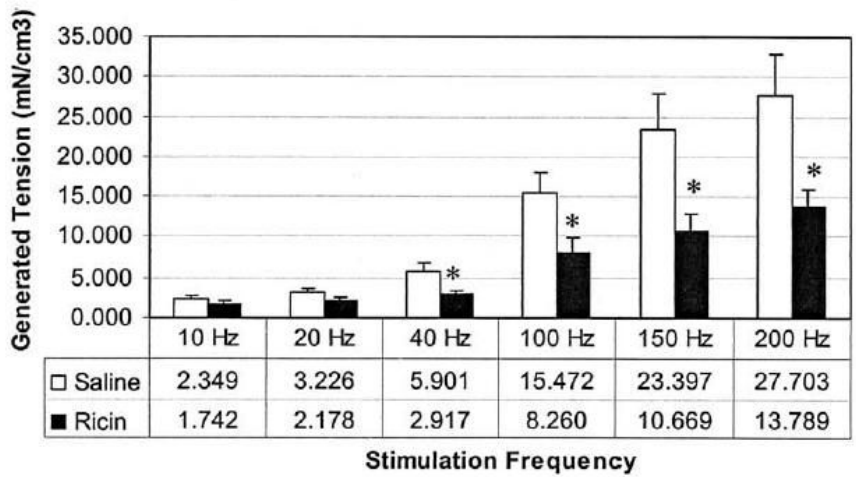

FIG 1.

Generated force (millinewtons/cubic centimeter $\left(\mathrm{mN} / \mathrm{cm}^{3}\right)$ ) with increasing stimulation frequency in ricin-mAb35-treated (Ricin) and saline-injected control (Saline) superior rectus muscles in rabbits at (A) 1 week $(\mathrm{n}=3)$; (B) 6 weeks $(\mathrm{n}=5)$; and $(\mathbf{C}) 12$ weeks $(\mathrm{n}=8)$ postinjection (250 millisecond train duration; $\geq 5 \mathrm{~V})$. $* P \leq .05$. 
Fatigue Testing - 12 weeks

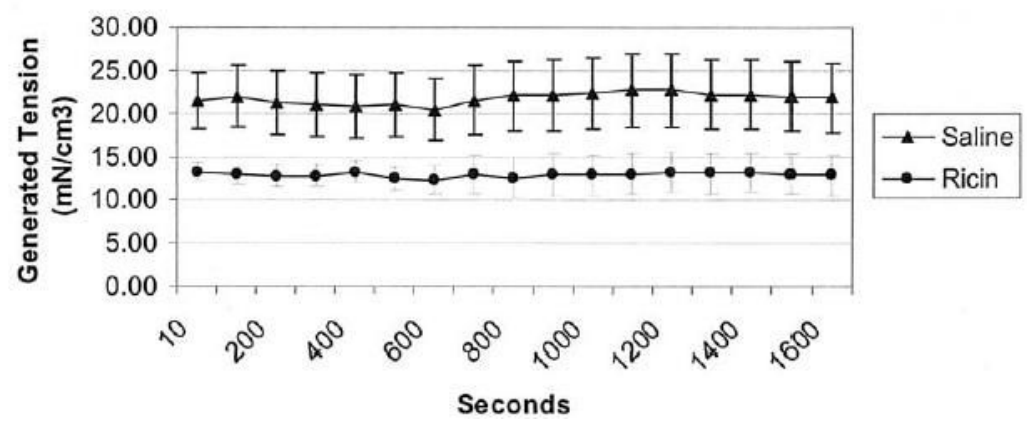

FIG 2.

Generated force (millinewtons/cubic centimeter $\left(\mathrm{mN} / \mathrm{cm}^{3}\right)$ ) during fatigue testing of ricinmAb35-treated (Ricin) and saline-injected control (Saline) superior rectus muscles in rabbits at 12 weeks postinjection $(100 \mathrm{~Hz} ; 250$ millisecond train duration; $\leq 5 \mathrm{~V})$. Similar results were seen at the 1-week and 6-week postinjection intervals. 

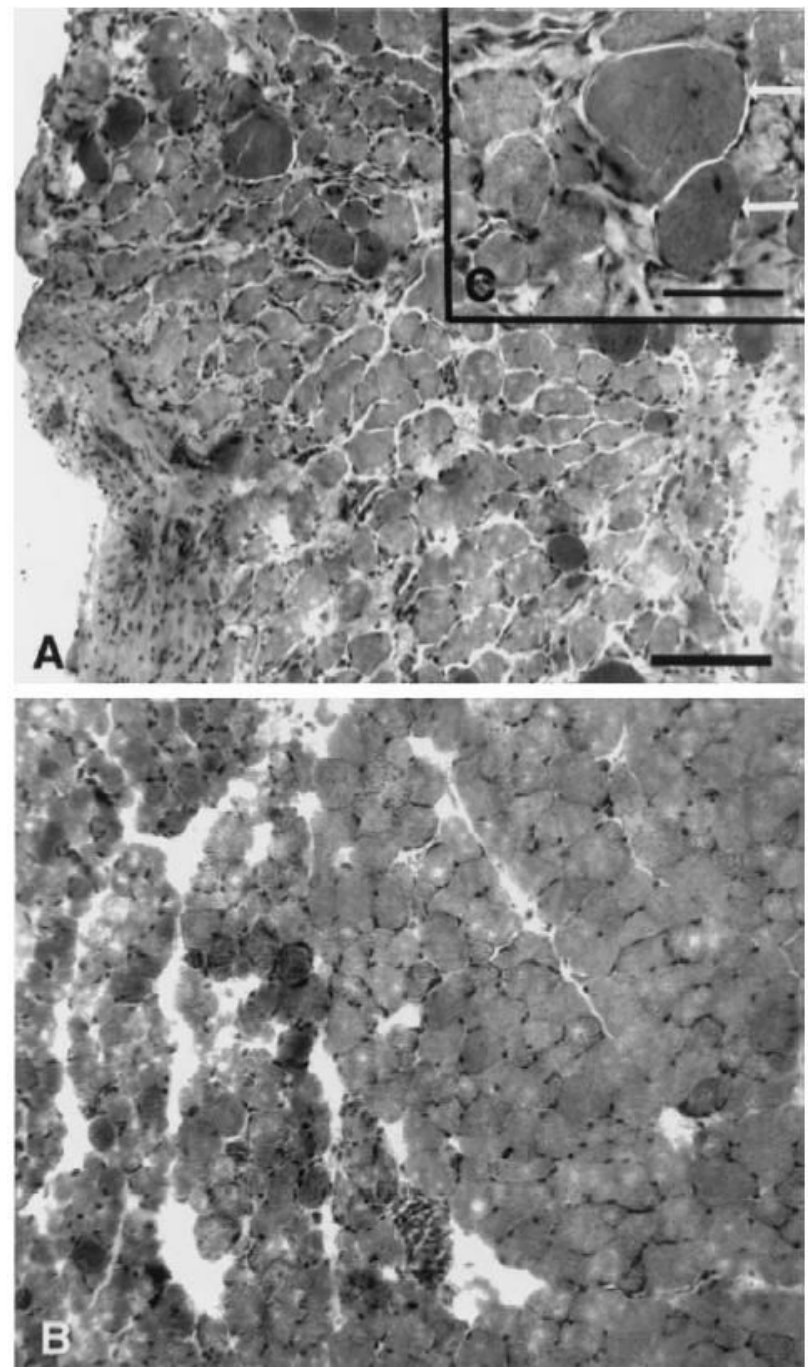

FIG 3.

A, Superior rectus muscle 6 weeks after ricin-mAb35 injection and physiological assessment of muscle force, stained with hematoxylin and eosin (bar $=100$ microns). B, Saline-injected control from the contralateral side of the same rabbit as A. C, Inset from A, showing magnified view of hypertrophic muscle fibers with central nuclei indicating active muscle fiber regeneration (white arrows; bar $=50$ microns). 
TABLE 1

Single-twitch tension $\left(\mathrm{mN} / \mathrm{cm}^{3} \pm \mathrm{SEM}\right)^{*}$ measured at optimal preload $(0.4$ milliseconds, $\geq 5 \mathrm{~V})$ at 1,6 , and 12 weeks following injection

\begin{tabular}{|c|c|c|c|c|}
\hline Post- injection interval (wks) & Saline & Ricin- mAb35 & $\%$ control & Pvalue \\
\hline $\begin{array}{l}1(n=3) \\
6(n=5) \\
12(n=8)\end{array}$ & $\begin{array}{l}3.48 \pm 1.17 \\
2.61 \pm 0.81 \\
2.93 \pm 0.60\end{array}$ & $\begin{array}{l}1.97 \pm 0.61 \\
1.13 \pm 0.16 \\
1.96 \pm 0.30\end{array}$ & $\begin{array}{l}56.6 \\
43.3 \\
66.9\end{array}$ & $\begin{array}{l}.169 \\
.121 \\
.038\end{array}$ \\
\hline
\end{tabular}

$\mathrm{mN} / \mathrm{cm}^{3}$, millinewtons/cubic centimeter. 
TABLE 2

Tetanic tension development $\left(\mathrm{mN} / \mathrm{cm}^{3} \pm \mathrm{SEM}\right)^{*}$ measured at optimal preload $(150 \mathrm{~Hz}, 250$ milliseconds, $\geq 5 \mathrm{~V})$ at 1,6 , and 12 weeks following injection

\begin{tabular}{llccc}
\hline Post- injection interval (wks) & Saline & Ricin-mAb35 & \% control & PValue \\
\hline $1(\mathrm{n}=3)$ & $37.17 \pm 14.72$ & $27.38 \pm 13.04$ & 73.7 & .119 \\
$6(\mathrm{n}=5)$ & $21.83 \pm 3.24$ & $9.04 \pm 1.44$ & .007 \\
$12(\mathrm{n}=8)$ & $23.40 \pm 4.45$ & $10.67 \pm 2.12$ & 45.4 & .022 \\
\hline
\end{tabular}

${ }^{*} \mathrm{mN} / \mathrm{cm}^{3}$, millinewtons/cubic centimeter. 